\title{
A Novel Approach to Solve Quasiexactly Solvable Pauli Equation
}

\author{
Ramazan Koç, Eser Olğar, and Haydar Mutaf \\ Department of Engineering Physics, Gaziantep University, 27310 Gaziantep, Turkey \\ Correspondence should be addressed to Eser Olğar; olgar@gantep.edu.tr
}

Received 9 October 2014; Accepted 6 December 2014

Academic Editor: Nikos Mastorakis

Copyright (C) 2015 Ramazan Koç et al. This is an open access article distributed under the Creative Commons Attribution License, which permits unrestricted use, distribution, and reproduction in any medium, provided the original work is properly cited.

\begin{abstract}
The spectra for some specific forms of external magnetic fields in Pauli equation are obtained in the framework of the asymptotic iteration method (AIM). AIM is applied to find the solution of Pauli equation. When the method is applied to quasiexactly solvable systems, it not only easily gives the corresponding spectrum, but also produces accurate results for the eigenvalues of the system having $s l(2)$ symmetry.
\end{abstract}

\section{Introduction}

It is well known that motion of the charged particles in electromagnetic fields described by Pauli equation is one of the fascinating phenomena in modern physics, providing a general approach to understanding the properties of electrons. The Hamiltonian for this system when $\hbar=e=2 m_{e}=$ $c=1$ is

$$
H=\left(p_{x}+A_{x}\right)^{2}+\left(p_{y}+A_{y}\right)^{2}+\frac{g}{2}(\nabla \times \mathbf{A})_{z} \sigma_{z},
$$

where $p_{x}, p_{y}$ are the momentum operators, $g=2$ is the gyromagnetic ratio, and $\mathbf{A}$ and $\sigma_{z}$ are the vector potential of the electromagnetic field and the Pauli matrix, respectively. The Pauli equation has been solved exactly for uniform magnetic field $B_{x}=B_{y}=0, B_{z}=B_{0}$, leading the Landau levels. Moreover, it was proved in [1] that for any general magnetic field $B_{z}=B(x, y)$ perpendicular to the $x y$ plane, the ground state is solved exactly as determined in [2-4]. The general result of [1] can be viewed as a very special case of the recently discovered QES systems that only a finite spectrum to be solved with algebraic methods [5-9]. The Pauli equation has been solved in a quasiexactly solvable model [10] for possible (nonuniform) magnetic fields [11]. If not impossible, it seems difficult to find other forms of the magnetic field such that the Pauli equation could be exactly solved. Therefore, the forms of magnetic fields, for which Pauli equation could be exactly solved, are indeed rather rare.

Therefore, a natural question arising at this point is can the asymptotic iteration method be applied to these systems? The answer to this question is the main topic of this paper. An asymptotic iteration technique which improves both analytical and numerical solutions of the problems has been introduced by Çiftçi et al. [12]. Afterwards, it has been developed for many physical systems in order to obtain the whole spectra $[13,14]$. It has been also suggested to solve the Schrödinger equation and developed for some quantum optical systems [15]. Later, a considerable attention has been paid to AIM [12, 16-19]. The results obtained by this method fit with the solutions of many exactly solvable differential equations in the form of the Schrödinger equation. It also gives accurate result for the nonexactly solvable Schrödinger equation such as sextic oscillator, cubic oscillator, and deformed coulomb potential, which are important in applications to many problems in physics. Although AIM has been applied to solve the Schrödinger equation, its application to the solution of the Pauli equation is still missing. It is well known that the AIM is very powerful in describing many physical problems. It improves both analytical and numerical solutions and also it is useful for understanding the nature of physical structures.

One can show that the termination relation $\Delta_{n}(x)=0$ in the corresponding method reflects effectively the peculiar properties of the functions $\lambda_{0}$ and $s_{0}$. After appropriate change of the variable, their hidden polynomial nature can be revealed. On these polynomials of finite order, the finitedimensional representations of the hidden $\operatorname{sl}(2, R)$ algebraic structure underlying the quasiexact solvability are realized. Also with this hidden polynomial structure, the nonlinear supersymmetry discussed in [10] can be related. 


\section{Formalism of the Asymptotic Iteration Method}

The AIM is proposed to solve the second-order differential equations and details can be found in [12]. In this section we systematically apply the method to obtain eigenvalues of the Pauli equation for various nonuniform magnetic fields.

Consider the following second-order differential equation:

$$
y^{\prime \prime}=\lambda_{0} y^{\prime}+s_{0} y
$$

where $\lambda_{0} \neq 0$ and $\lambda_{0}, s_{0}$ are functions defined in $C_{\infty}(a, b)$ and they have sufficiently many continuous derivatives. $y^{\prime}$ and $y^{\prime \prime}$ denote derivatives of $y$ with respect to $x$. It is easy to show that $(n+2)$ th derivative of the function $y$ can be written as

$$
y^{(n+2)}=\lambda_{n} y^{\prime}+s_{n} y,
$$

where $\lambda_{n}$ and $s_{n}$ are given by the recurrence relations

$$
\lambda_{n}=\lambda_{n-1}^{\prime}+s_{n-1}+\lambda_{n-1} \lambda_{0}, \quad s_{n}=s_{n-1}^{\prime}+\lambda_{n-1} s_{0} .
$$

If we have, for sufficiently large $n$,

$$
\frac{s_{n}}{\lambda_{n}}=\frac{s_{n-1}}{\lambda_{n-1}}=\alpha_{n}(x),
$$

then the solution of (2) can be written as follows:

$$
\begin{aligned}
y= & \exp \left(-\int^{x} \alpha d t\right) \\
& \cdot\left[C_{1}+C_{2} \int^{x} \exp \left(\int^{s}\left(\lambda_{0}+2 \alpha\right) d t\right) d s\right],
\end{aligned}
$$

where $C_{1}$ and $C_{2}$ are the integration constants. In calculating the parameters in (4), when $n=0$, we take the initial conditions as $\lambda_{-1}=1$ and $s_{-1}=0$ [14] and the termination condition $\Delta_{n}(x)$ is $\Delta_{n}(x)=0$ for

$$
\Delta_{n}(x)=\lambda_{n}(x) s_{n-1}(x)-\lambda_{n-1}(x) s_{n}(x) .
$$

Before going further we note that, in this paper, we will determine magnetic fields that lead to QES solvable potential forms in Pauli equation. We consider the magnetic fields only in asymmetric gauge. Solution of the Pauli equation including nonuniform magnetic fields has recently been studied in the framework of the QES systems [10, 11]. Ho and Roy presented a general procedure for determining possible (nonuniform) magnetic fields such that the Pauli equation becomes QES with an underlying $s l(2)$ symmetry. But our paper includes a part of the motivation those QES systems which can be solved by using AIM and it gives the whole spectrum with accurate results.

The following section includes the solution of Pauli Equation within the framework of the AIM.

\section{Magnetic Field}

In this paper, we deal with magnetic field in asymmetric gauge with vector potentials as in [11]

$$
A_{x}(x, y)=0, \quad A_{y}(x, y)=-\bar{W}(x) .
$$

In this case components of the magnetic field $\mathbf{B}$ with asymmetric gauge are given by

$$
B_{x}=B_{y}=0, \quad B_{z}=B(x)=-\bar{W}^{\prime}(x),
$$

where $\bar{W}(x)$ is an arbitrary function of $x$ and $\bar{W}^{\prime}(x)=$ $d \bar{W}(x) / d x$. The Pauli Hamiltonian then takes the form

$$
H=p_{x}^{2}+\left(p_{y}-\bar{W}(x)\right)^{2}-\bar{W}^{\prime}(x) \sigma_{z}
$$

The variables $x$ and $y$ can be separated by introducing the wavefunction

$$
\psi(x, y)=e^{-i k y} \phi(x) .
$$

Here $k(-\infty<k<\infty)$ are the eigenvalues of $p_{y}$, and $\phi(x)=$ $\left(\begin{array}{l}\phi_{+}(x) \\ \phi_{-}(x)\end{array}\right)$ is a two-component function of $x$. Then the Hamiltonian, $H$, can be written as

$$
H_{\mp}=-\frac{d^{2}}{d x^{2}}+(\bar{W}(x)+k)^{2} \mp \bar{W}^{\prime}(x) .
$$

It is obvious that the Hamiltonian has been written in the one-dimensional form of the Schrödinger equation with the potential. This final form is the SUSY form of the Pauli equation with the superpotential $W(x)=\bar{W}(x)+k$.

Now, our aim is to determine the magnetic fields that lead to the QES potential forms. By defining the magnetic fields in appropriate forms that construct QES potentials, we will try to apply AIM to the resultant Hamiltonian.

3.1. Class I. In order to obtain the Class I potential, the corresponding magnetic field can be defined as

$$
B=-\bar{W}^{\prime}(x)=-a e^{-\alpha x}-b e^{\alpha x} .
$$

In this case we can write the following eigenvalue problem $H \psi(x)=E \psi(x)$ form (12) for $H_{-}$part that satisfies the Schrödinger equation under the normalizable zero energy state condition

$$
\left[-\frac{d^{2}}{d x^{2}}+V(x)\right] \psi(x)=E \psi(x),
$$

where

$$
\begin{aligned}
V(x)=\frac{1}{\alpha^{2}}[ & a^{2} \exp (-2 \alpha x)+b^{2} \exp (2 \alpha x) \\
& -a\left(2 k \alpha+\alpha^{2}\right) \exp (-\alpha x) \\
& \left.+b\left(2 k \alpha-\alpha^{2}\right) \exp (\alpha x)+k^{2} \alpha^{2}-2 a b\right]
\end{aligned}
$$

is the Class I potential according to Turbiner's classification in [6]. After redefining the variable as $z=\exp (\alpha x)$ and using $\psi=z^{-1 / 2} \chi(z)$ in (14), we get the following differential equation:

$$
\left[-\frac{d^{2}}{d z^{2}}+\frac{A^{2}}{z^{4}}+B^{2}-\frac{C}{z^{3}}-\frac{D}{z}+\frac{\gamma(\gamma+1)}{z^{2}}\right] \chi(z)=0,
$$


TABLE 1: Type 1 energy values when $A=B=1$.

\begin{tabular}{|c|c|c|c|c|c|}
\hline$n$ & $u=-2 n B$ & $v=-2 A$ & $s$ & $E_{n s}$ & $E_{n s k}$ \\
\hline 0 & 0 & -2 & -1 & 4 & Infinity \\
\hline \multirow{2}{*}{1} & \multirow{2}{*}{-2} & \multirow{2}{*}{-2} & -2 & 2 & 0 \\
\hline & & & -0.5 & 5 & -8 \\
\hline \multirow{3}{*}{2} & \multirow{3}{*}{-4} & \multirow{3}{*}{-2} & -2.63571 & -0.54284 & -51.3481 \\
\hline & & & -1.71698 & 3.13208 & -0.790868 \\
\hline & & & -0.14731 & 9.41074 & 0.997333 \\
\hline \multirow{4}{*}{3} & \multirow{4}{*}{-6} & \multirow{4}{*}{-2} & -3.12512 & -2.75072 & -11.7596 \\
\hline & & & $-5 / 2$ & 1.0 & -1.77778 \\
\hline & & & -1.51563 & 6.90622 & 0.627819 \\
\hline & & & 0.14075 & 16.8445 & 0.998845 \\
\hline \multirow{5}{*}{4} & \multirow{5}{*}{-8} & \multirow{5}{*}{-2} & -3.54136 & -4.33088 & -4.89446 \\
\hline & & & -3.10868 & -0.86944 & -1.70161 \\
\hline & & & -2.39350 & 4.852 & 0.15676 \\
\hline & & & -1.34675 & 13.226 & 0.864101 \\
\hline & & & 0.390287 & 27.1223 & 0.994757 \\
\hline \multirow{6}{*}{5} & \multirow{6}{*}{-10} & \multirow{6}{*}{-2} & -3.92981 & -5.2981 & -2.60348 \\
\hline & & & -3.61617 & -2.1617 & -1.30116 \\
\hline & & & -3.07259 & 3.2741 & -0.101646 \\
\hline & & & -2.29765 & 11.0235 & 0.614865 \\
\hline & & & -1.1973 & 22.027 & 0.937851 \\
\hline & & & 0.613514 & 0.1351 & 0.991394 \\
\hline
\end{tabular}

where $A=a / \alpha^{2}, B=b / \alpha^{2}, C=a(2 k+\alpha) / \alpha^{3}, D=b(-2 k+$ $\alpha) / \alpha^{3}$, and $\gamma(\gamma+1)=\left(\left(\alpha^{2}\left(k^{2}-E\right)-2 a b\right) / \alpha^{4}\right)-1 / 4$. Before applying AIM to (16), we have to obtain the asymptotic form for $\chi(z)$. It is easy to see that when $z \rightarrow 0$ and $z \rightarrow \infty$, $\chi(z) \sim z^{s+1} \exp (-A / z-B z)$, and after substituting $\chi(z)=$ $z^{s+1} \exp (-A / z-B z) f(z)$ into (16), we obtain

$$
f^{\prime \prime}=2\left(B-\frac{s+1}{z}-\frac{A}{z^{2}}\right) f^{\prime}+\left(\frac{u}{z}-\frac{v}{z^{3}}\right) f,
$$

where $u=2(s+1) B-D, v=2 s A+C$, and $s(s+1)=\gamma(\gamma+$ $1)+2 B A$. Now, we can apply AIM to this final equation with $\lambda_{0}=2\left(B-(s+1) / z-A / z^{2}\right)$ and $s_{0}=(u / z)-\left((2 s A+C) / z^{3}\right)$ and we will show that (17) has exact solutions under some constraints on the parameters $A, B, C$, and $D$.

If we apply AIM quantization condition, that is, from (4) by substituting $\lambda_{0}$ and $s_{0}$, in order to obtain polynomial solutions we see that the parameters must be equal to $u=$ $-2 n B, v=-2 A$ and $s$ values are given in Table 1; we will show the results for type 1 and $a=b=\alpha=1$ for simplicity. Note that there are two types of solutions that give us the polynomials, for example, for $n=2, v=-2 A$ (as given above), and $v=(6 / B)((s+2)(2 s+3)-A B)$. If we use the first value of $v$ we get the results given in Table 1, but one can use the second value of $s$ and get different results which give us the polynomials. We will not give this second situation here because of complication on the calculations. In this paper, we use only $v=-2 A$ type solution. If we use $u=2(s+1) B-D, v=$ $2 s A+C, s(s+1)=\gamma(\gamma+1)+2 B A, u=-2 n B$, and $v=-2 A$, we have $D=2(n+s+1) B$ and $C=-2(s+1) A$. If we go back to
TABLE 2: Type 2 energy values when $A=B=1$.

\begin{tabular}{|c|c|c|c|}
\hline$n$ & $u=-2 n B$ & $v$ & $s$ \\
\hline 0 & 0 & -2 & -1.0 \\
\hline \multirow{2}{*}{1} & \multirow{2}{*}{-2} & \multirow{2}{*}{-2} & -2.0 \\
\hline & & & -0.5 \\
\hline \multirow{5}{*}{2} & \multirow{5}{*}{-4} & \multirow{5}{*}{$6(s+2)(2 s+3)$} & -2.5 \\
\hline & & & -2.22871 \\
\hline & & & -2.0 \\
\hline & & & -1.27129 \\
\hline & & & -1.0 \\
\hline \multirow{9}{*}{3} & \multirow{9}{*}{-6} & & 3.13571 \\
\hline & & & -2.80396 \\
\hline & & & -2.63188 \\
\hline & & & -2.5 \\
\hline & & $4\left(12 s^{3}+72 s^{2}+125 s+54\right)$ & -2.21698 \\
\hline & & $10 s+23$ & -2.11304 \\
\hline & & & -1.86812 \\
\hline & & & -1.08301 \\
\hline & & & -0.647315 \\
\hline
\end{tabular}

definitions of $A, B, C$, and $D$, we find the following expression for the energy as follows:

$$
E_{n s}=\frac{n(1+n+2 s) \alpha^{4}+2 a b\left(1+\alpha^{2}\right)}{\alpha^{2}} .
$$

If the parameters in (18) are mapped to the parameters in energy values in $[10,11]$, the energy values can be in accord. In Table 1, we will show the results for type 1 and $a=b=\alpha=1$ for simplicity. Similarly, in Table 2, we will show the results of different values of $s$ for $A=B=1$ for type 2 solution.

3.2. Class IX. Let us assume that the magnetic field takes the form of

$$
B=-\bar{W}^{\prime}(x)=a x^{-3}+b x^{-2} .
$$

With this magnetic field, we can write the following eigenvalue problem for $H_{-}$after replacing $x$ by $z$ :

$$
\left[-\frac{d^{2}}{d z^{2}}+\frac{A^{2}}{z^{4}}+B^{2}-\frac{C}{z^{3}}-\frac{D}{z}+\frac{F}{z^{2}}\right] \chi(z)=0,
$$

where $A=a, B=\sqrt{k^{2}-E}, C=2 a(1+b), D=2 b k$, and $F=b(b+1)+2 a k$. This potential form is the Class IX potential form. By applying the same procedure performed in the previous potential form, when $z \rightarrow 0$ and $z \rightarrow \infty$, $\chi(z) \sim z^{s+1} \exp (-A / z-B z)$, and after substituting $\chi(z)=$ $z^{s+1} \exp (-A / z-B z) f(y)$ into (20), we have

$$
f^{\prime \prime}=2\left(B-\frac{s+1}{z}-\frac{A}{z^{2}}\right) f^{\prime}+\left(\frac{u}{z}-\frac{v}{z^{3}}\right) f .
$$

Here $u=2(s+1) B-D, v=2 s A+C$, and $s(s+1)=F+2 B A$. In this form of differential equation, if we apply AIM to this final 
equation with $\lambda_{0}=2\left(B-(s+1) / z-A / z^{2}\right)$ and $s_{0}=u / z-(2 s A+$ $C) / z^{3}$, we will obtain the exact solutions of (20) under some constraints on the parameters $A, B, C$, and $D$. It can be easily seen that the solutions of this form of potential are equal to the solution of the first example except for definitions of $A, B$, $C$, and $D$. In this type of potential, the definitions of $E_{n s k}$ and $b$ for the type 1 solution can be obtained as below:

$$
\begin{aligned}
b & =-s, \\
E_{n s k} & =k^{2}-\left(\frac{-k s}{n+s+1}\right)^{2} .
\end{aligned}
$$

\section{Results and Discussion}

The energy obtained for Class IX potential in the form of parameters $A, B, C, D$, and $F$ agrees with those in $[20,21]$. Similar to the Class I potential, the values obtained for the second type solution in Table 2 are valid for this potential. The energy in (22) depends only on $k, s$, and $n$. For simplicity, the energy values for $k=1$ are also tabulated in Table 1 .

Similar to the procedure presented in this study, it is possible to obtain the same solutions for other class potentials described by Turbiner [6] except for Class IV and Class V potentials. For these two potentials, an appropriate superpotential to construct the potentials has not been written because of the constant $k$ in the $\bar{W}(x)+k$. The other six classes satisfy the requirements of using AIM which is the differential equations conditions being transformable to a specific form in (2), with quite restrictive asymptotic conditions on the coefficient functions of $\alpha(x)$ in (5).

\section{Conclusions}

In this work, we have shown that the AIM method can also be used to obtain the QES energies obtained in $[10,11]$ by using the known forms of the magnetic field $\mathbf{B}$ with asymmetric gauge obtained in the same paper. We follow these corresponding studies and consider the magnetic field in that form. The procedure presented here makes full use of the close connection between QES systems and SUSY-like form. Additionally, an attempt to give the QES solution of the Pauli equation by using asymptotic iteration technique is presented. The application of AIM is considered for Class I and Class IX QES systems. By the same procedure, AIM can be applied to the other QES potentials in one dimension except for Class IV and Class V. The whole spectrum of the QES systems is presented.

Finally, we will try to apply this method to other QES potentials in all branches of physics.

\section{Conflict of Interests}

The authors declare that there is no conflict of interests regarding the publication of this paper.

\section{Acknowledgments}

The authors are grateful to the reviewer for her/his comments, which contributed to improving the quality of the paper. And the authors are also deeply indebted to Dr. Çiftçi for useful discussions. The research was supported by the Research Fund of Gaziantep University (BAP) and the Scientific and Technological Research Council of Turkey (TUBİTAK).

\section{References}

[1] Y. Aharonov and A. Casher, "Ground state of a spin-1/2 charged particle in a two-dimensional magnetic field," Physical Review A, vol. 19, no. 6, pp. 2461-2462, 1979.

[2] G. N. Stanciu, "Further exact solutions of the Dirac equation," Journal of Mathematical Physics, vol. 8, no. 10, pp. 2043-2047, 1967.

[3] F. Cooper, A. Khare, R. Musto, and A. Wipf, "Supersymmetry and the Dirac equation," Annals of Physics, vol. 187, no. 1, pp. $1-28,1988$.

[4] F. Cooper, A. Khare, and U. Sukhatme, "Supersymmetry and quantum mechanics," Physics Reports, vol. 251, no. 5-6, pp. 267385, 1995.

[5] A. V. Turbiner and A. G. Ushveridze, "Spectral singularities and quasi-exactly solvable quantal problem," Physics Letters A, vol. 126, no. 3, pp. 181-183, 1987.

[6] A. V. Turbiner, "Quasi-exactly-solvable problems and $s l(2)$ algebra," Communications in Mathematical Physics, vol. 118, no. 3, pp. 467-474, 1988.

[7] N. Kamran and P. J. Olver, "Lie algebras of differential operators and Lie-algebraic potentials," Journal of Mathematical Analysis and Applications, vol. 145, no. 2, pp. 342-356, 1990.

[8] M. A. Shifman, "New findings in quantum mechanics (partial algebraization of the spectral problem)," International Journal of Modern Physics A, vol. 4, no. 12, pp. 2897-2952, 1989.

[9] C.-L. Ho and V. R. Khalilov, "Planar dirac electron in coulomb and magnetic fields," Physical Review A: Atomic, Molecular, and Optical Physics, vol. 61, no. 3, 2000.

[10] S. M. Klishevich and M. S. Plyushchay, "Nonlinear supersymmetry, quantum anomaly and quasi-exactly solvable systems," Nuclear Physics B, vol. 606, no. 3, pp. 583-612, 2001.

[11] C.-L. Ho and P. Roy, "Quasi-exact solvability of the Pauli equation," Journal of Physics A: Mathematical and General, vol. 36, no. 16, pp. 4617-4628, 2003.

[12] H. Çiftçi, R. L. Hall, and N. Saad, "Asymptotic iteration method for eigenvalue problems," Journal of Physics A: Mathematical and General, vol. 36, no. 47, pp. 11807-11816, 2003.

[13] T. Barakat, K. Abodayeh, and A. Mukheimer, "The asymptotic iteration method for the angular spheroidal eigenvalues," Journal of Physics. A. Mathematical and General, vol. 38, no. 6, pp. 1299-1304, 2005.

[14] F. M. Fernández, "On an iteration method for eigenvalue problems," Journal of Physics. A. Mathematical and General, vol. 37, no. 23, pp. 6173-6180, 2004.

[15] I. Boztosun, M. Karakoc, F. Yasuk, and A. Durmus, "Asymptotic iteration method solutions to the relativistic Duffin-KemmerPetiau equation," Journal of Mathematical Physics, vol. 47, no. 6, Article ID 062301, 2006.

[16] A. J. Sous, "Solution for the eigenenergies of sextic anharmonic oscillator potential $V(x)=A_{6} x^{6}+A_{4} x^{4}+A_{2} x$," Modern Physics Letters $A$, vol. 21, no. 21, pp. 1675-1682, 2006.

[17] P. Amore and F. M. Fernández, "Comment on an application of the asymptotic iteration method to a perturbed Coulomb model," Journal of Physics, A: Mathematical and Theoretical, vol. 39, no. 33, pp. 10491-10497, 2006. 
[18] E. Olğar, "Exact solution of Klein. Gordon equation by asymptotic iteration method," Chinese Physics Letters, vol. 25, no. 6, pp. 1939-1942, 2008.

[19] Ö. Öztemel and E. Olğar, "An alternative solution of diatomic molecules," Central European Journal of Physics, vol. 12, no. 2, pp. 103-110, 2014.

[20] E. Papp, "Symmetry relationships between energy formulae and constraint conditions for interrelated superpositions of power potentials," Physics Letters A, vol. 157, no. 4-5, pp. 192-194, 1991.

[21] S.-H. Dong, Z.-Q. Ma, and G. Esposito, "Exact solutions of the Schrödinger equation with inverse-power potential," Foundations of Physics Letters, vol. 12, no. 5, pp. 465-474, 1999. 


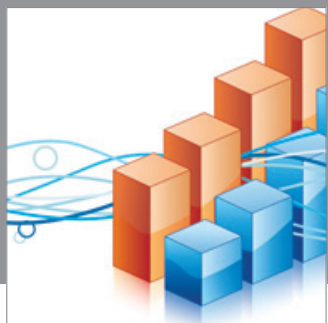

Advances in

Operations Research

mansans

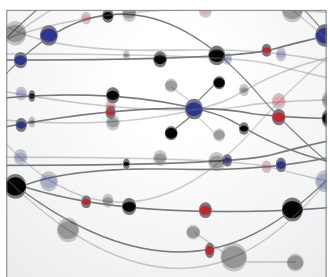

The Scientific World Journal
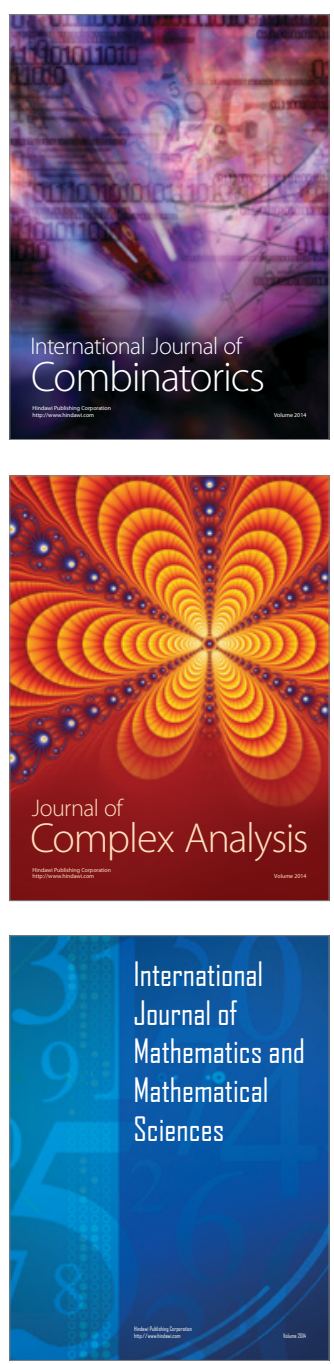
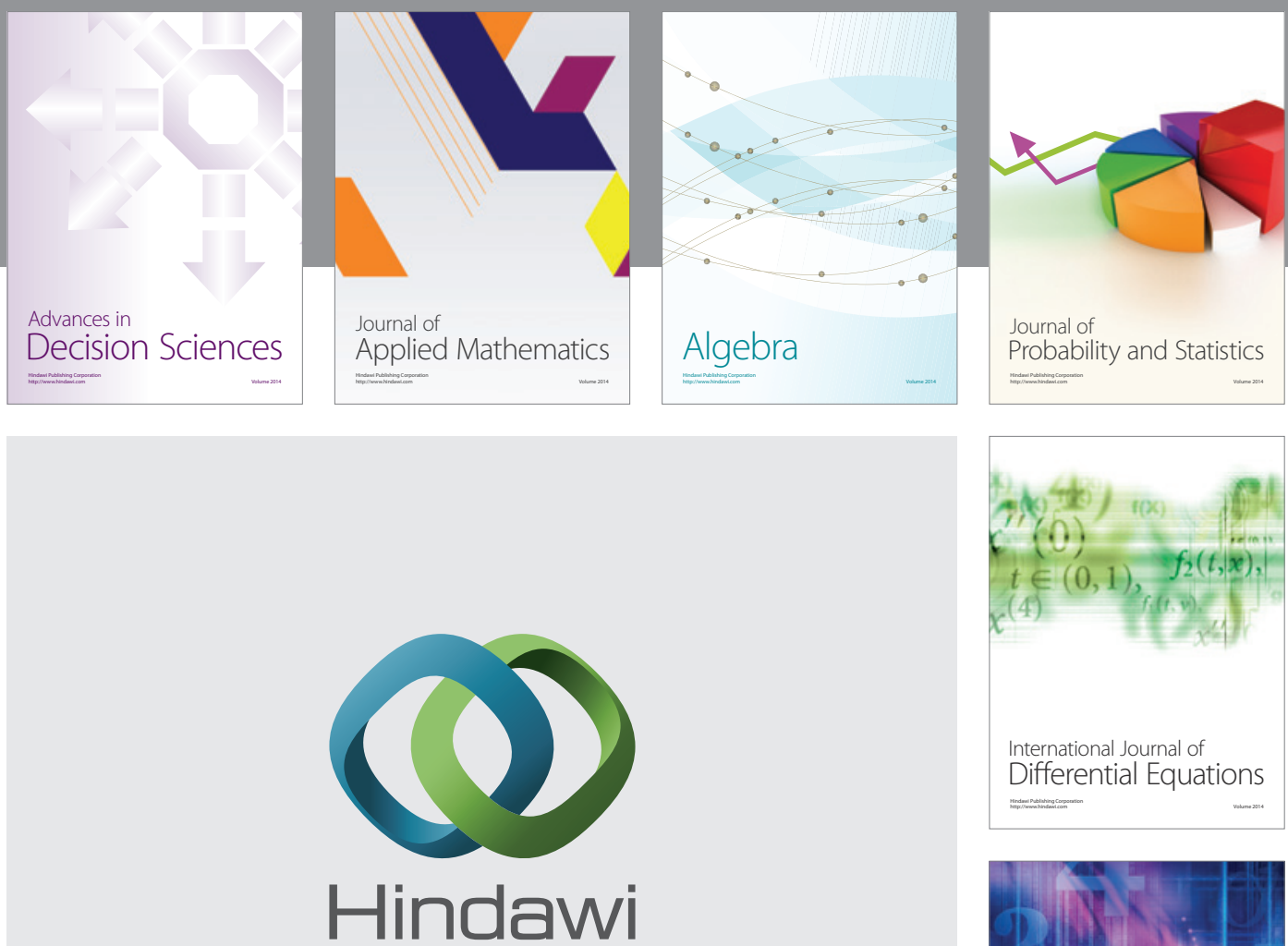

Submit your manuscripts at http://www.hindawi.com
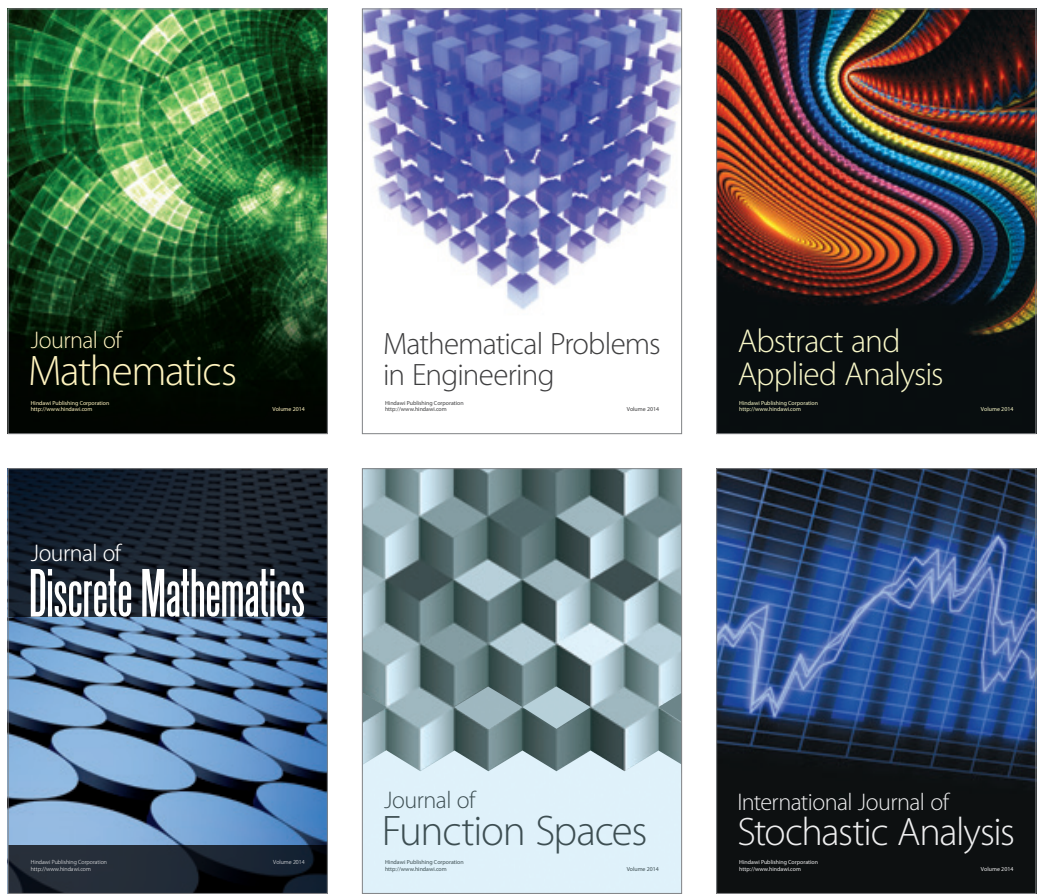

Journal of

Function Spaces

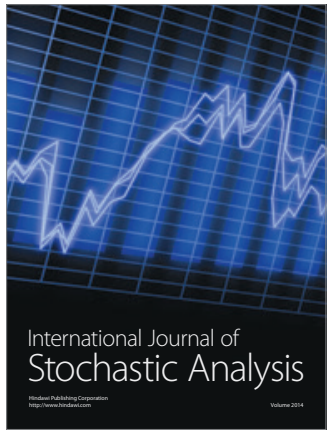

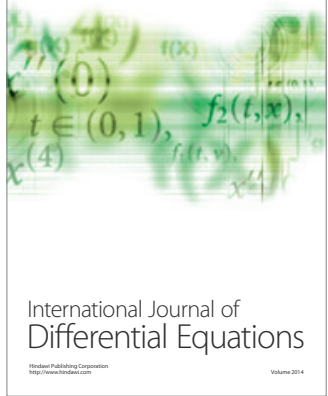
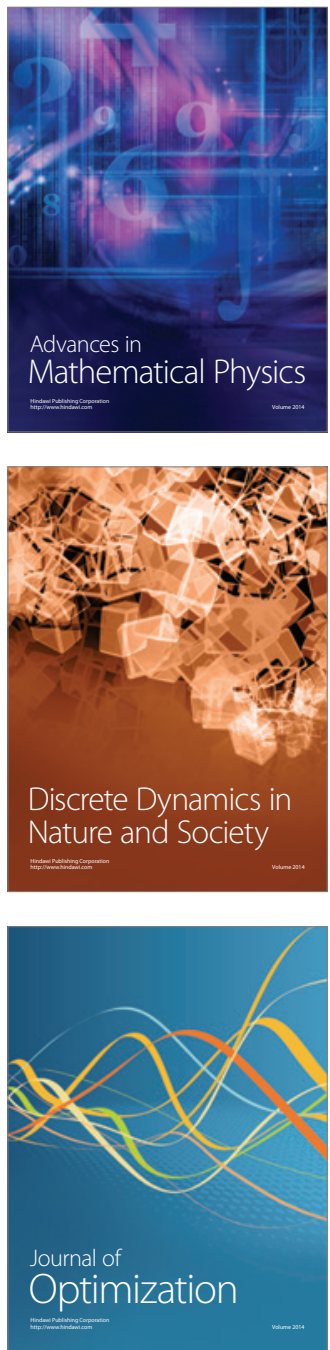